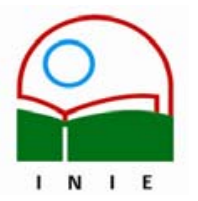

\title{
RASGOS DE SENTIDO DE VIDA DEL ENFOQUE DE RESILIENCIA EN PERSONAS MAYORES ENTRE LOS 65 Y 75 AÑOS
}

\author{
Flor Jiménez Segura ${ }^{1}$ \\ Irma Arguedas Negrini
}

\begin{abstract}
Resumen: La resiliencia es la capacidad para activar las fortalezas y proteger la integridad personal al enfrentar adversidades, construyendo a partir de éstas. En este estudio se identificaron los ocho rasgos de sentido de vida según el enfoque de resiliencia (Intereses especiales, Metas, Motivación para el logro, Aspiraciones educativas, Optimismo, Persistencia, Fe y Espiritualidad y Sentido de propósito), que han presentado personas adultas mayores de 65 a 75 años durante sus vidas. Los principios teórico-metodológicos utilizados son los del paradigma cualitativo. Se efectuaron entrevistas en profundidad a cuatro mujeres y cuatro hombres que pertenecen al grupo de Envejecimiento Integral de la Universidad de Costa Rica o a la Asociación Gerontológica Costarricense. Aunque la mayoría de las personas adultas mayores participantes en el estudio no identificaron metas claras en la actualidad, durante sus vidas contaron con las condiciones y herramientas personales que contribuyeron con el desarrollo de los rasgos de sentido de vida del enfoque de resiliencia.
\end{abstract}

Palabras clave: SENTIDO DE VIDA/ RESILIENCIA/ ORIENTACIÓN/BIENESTAR EN EL ADULTO MAYOR/

Abstract: The concept of resilience centers around the human capacity to rise from adversities and to be strengthened by them. Traits of the Sense of Purpose component of a resilience model (Special interests, Goal directedness, Achievement motivation, Educational aspirations, Hopefulness, Persistence, Faith and Spirituality, and Meaningfulness) were identified in older persons 65 to 75 years of age. In-depth interviews were conducted with four women and men who participate in programs that promote healthy development in older adults. Although most of the persons interviewed had difficulty specifying present goals, during their lives and currently they have had the environmental and personal conditions for the strengthening of the traits identified with the presence of a sense of purpose.

Key words: SENSE OF PURPOSE/ RESILIENCE/ COUNSELING/WELL-BEING IN OLDER ADULT/

\section{INTRODUCCIÓN}

Se realizan alrededor del mundo numerosas investigaciones acerca de cómo aumentar la longevidad y mejorar la calidad de vida de los seres humanos. No obstante, además de conocimientos y una sociedad más instruida, necesitamos que las personas mayores sean conscientes de su proceso de envejecimiento, de planear y anticipar su vejez. Para ello deberían reconocer temprano sus fortalezas y clarificar el significado que dan a su propia vida (Bonilla y Mata, 1998).

\footnotetext{
${ }^{1}$ Magister Scientae en Gerontología, Universidad de Costa Rica. Docente de la Escuela de Orientación y Educación Especial y de la Maestría en Gerontología de la Universidad de Costa Rica. Coordinadora del Programa de Orientación de la Universidad Estatal a Distancia. Varias publicaciones especializadas. Correo electrónico: fijimene@cariari.ucr.ac.cr

2 Master of Science en Orientación Escolar, Department of Applied Behavioral Sciences in Education, Universidad Estatal de Oklahoma, E.E.U.U. Docente de la Escuela de Orientación y Educación Especial de la Universidad de Costa Rica.

Correo electrónico: quarco2000@yahoo.com
}

Artículo recibido: 26 de julio, 2004

Aprobado: 26 de agosto, 2004 
Los individuos involucrados en su existencia son más vitales, saben dar continuidad a los objetivos planteados y son responsables, lo cual les da sentido de vida. Cuanto más pronto se aclaren estos significados, menos complicado será definir una intencionalidad o visión hacia el futuro, y a su vez se posibilitará la adecuada definición de aquello que se considera esencial y relevante en la vida (Frankl, 1999). Para la persona mayor, esta capacidad de valorar la vida le facilita mantener la autoestima y definir su nivel de autorrealización. Las personas con claridad de sentido, pueden evaluar más objetivamente los resultados de sus vidas (Bonilla y Rapso, 1997).

Todo lo anterior fortalece al adulto mayor, pues le da una sensación de bienestar y control sobre su vida, le ayuda a mantener su motivación a pesar del estrés en su etapa final y le permite reconciliarse consigo mismo y con el mundo. Las personas que logran aclarar el sentido que para ellas tiene la vida, son capaces de adecuar el "guión básico" de su existencia, de modificar relaciones, reorganizar su mundo laboral, enfrentar adversidades y hasta de reconstruir su escala de valores, incorporando nuevas creencias, objetivos y metas. Por el contrario, los que no logran clarificar su propósito de vida durante estos últimos años se sentirán desesperados, desmoralizados, y manifestarán miedo a morir (Erikson, 1970).

El significado que se da a la vida cambia de acuerdo con las distintas etapas que se van superando. Las personas son capaces de encontrar un sentido en su vida independientemente del sexo, la edad, el cociente intelectual, la educación recibida, el medio ambiente y ser creyente o no. Sin embargo, es importante no subestimar la influencia negativa de las condiciones de pobreza en el desarrollo personal de los individuos (Noblejas, 2000; Kotliarenco, 1995).

No solo las causas nobles y placenteras dan sentido de vida; a veces lo dan también el dolor y el sufrimiento. De ahí que, a menudo, la claridad de sentido ayude a observar los errores como esfuerzos, los problemas como desafíos y devele rasgos de empatía, altruismo, excelencia, alegría, autonomía y serenidad en los mayores, que los convierten en personas sobrevivientes (Fabry, 1992).

El presente estudio pretende identificar cuáles rasgos de sentido de vida (intereses especiales, metas, motivación para el logro, persistencia, aspiraciones educativas, optimismo, fe y espiritualidad y sentido de propósito), propuestos por el enfoque de resiliencia, se han presentado en las personas mayores de 65 a 75 años participantes, durante sus vidas. El enfoque de resiliencia plantea que una persona es resiliente si tiene una actitud de vivir situaciones adversas sin dañarse, si puede preservar la integridad en 
circunstancias difíciles y reaccionar positivamente, desarrollándose en medio de las dificultades (Wolin y Wolin 1993).

\section{MARCO TEÓRICO}

\subsection{LA VEJEZ}

Cada etapa de la vida nos brinda oportunidades para transformaciones creativas y crecimiento a partir de los retos que se tengan que enfrentar (Viorst, 1990). La vejez o etapa de la integración es una de ellas. Si se puede predecir quiénes van a navegar por las transiciones de la vida más exitosamente, hay más posibilidades de promocionar el envejecimiento saludable (Ramsey y Blieszner, 1999). Los factores que influencian la forma en que se da la adaptación a los múltiples cambios de estos años son una combinación de rasgos personales, estilos de vida y factores ambientales. Corey y Corey (1997) consideran que a esto hay que agregar las creencias que tenga el individuo acerca del cambio, que es una realidad con la que se tiene que lidiar durante toda la adultez. De acuerdo con ellos, la apertura al cambio es un factor de peso en los adultos mayores que gozan de bienestar y el considerar que en su mayoría no tienen esta apertura está basado más en un estereotipo que en los hechos.

Según Thorson (1995), los adultos que tienen niveles de adaptación más maduros tienden a mantenerlos dentro de un marco bastante estable a lo largo de la vida adulta y hasta bien entrados en la vejez. Sin embargo, cualquier adulto sano puede pasar por períodos de inconsistencia en cuanto a la presencia de las características que promueven el bienestar; lo que es determinante es el balance que se logre alcanzar (Viorst,1992). Las tres defensas o mecanismos maduros principales que determinan el nivel de adaptación de la persona son: la anticipación, la sublimación y la supresión. Sheehy (1987) aclara que con estas estrategias la persona no niega la existencia de realidades desagradables; todo lo contrario, las reconoce y utiliza las estrategias para aceptarlas. La anticipación es la capacidad de planear para el futuro; reduce la ansiedad y aumenta la sensación de control interno. La importancia que Thorson (1995) observa de adquirir o conservar el control interno que el adulto saludable logra con la madurez, es que éste constituye una de las mejores maneras de mantener la continuidad de roles y mecanismos adaptativos en la vejez. La sublimación consiste en canalizar las emociones de formas aceptables, tales como la expresión artística o la dedicación al trabajo. Cuando la persona toma la decisión consciente de posponer el enfrentamiento con un conflicto para evitar actos inapropiados o 
autodestructivos, está practicando la supresión. Es esto lo que permite seguir funcionando a pesar de molestias y dolores.

Otras defensas maduras incluyen conductas atractivas y favorecedoras de las relaciones interpersonales satisfactorias tales como el humor, la generosidad a pesar de tener problemas propios y el levantarse de las pérdidas. Vaillant (2000) destaca el altruismo como uno de los mecanismos maduros o saludables. El continuar dando a otros resistiéndose a caer en la autoabsorción contribuye, de acuerdo con Sheehy (1987), al bienestar, al representar el valor de una persona que aún se siente responsable por otros con más necesidad y al contribuir con el enfoque optimista de que el futuro puede verse con esperanza. Esta autora encontró dentro de las características de personas adultas con alto nivel de bienestar, que los hombres reportaban sentirse más a gusto con la intimidad y las mujeres con la ambición, lo que indica que el adulto más satisfecho ha incorporado la característica más asociada con el género opuesto, es decir, ha trascendido algunos estereotipos.

\subsection{ENFOQUE DE RESILIENCIA}

La resiliencia, o capacidad para minimizar los efectos negativos de la adversidad y maximizar los positivos, está determinada por múltiples factores. De acuerdo con Werner y Smith (2001), resulta de una interacción entre factores protectores, tanto inherentes al individuo como externos, y los riesgos que se dan en la vida de las personas. Tal y como lo resume Kotliarenco (1995), los procesos de vulnerabilidad / protección, conducen a beneficios o desórdenes, dependiendo de su interacción con las variables de riesgo. Los patrones de adaptación del individuo también determinan si los desafíos tienen influencia positiva o negativa. Se ha argumentado que enfrentar situaciones difíciles puede ser constructivo y utilizarse para fortalecer habilidades, desarrollar la persistencia y disfrutar estados de emoción positiva como la satisfacción que dan las actividades creativas (Maslow, 1976; Seligman, 1995). El estudio de Vaillant (2000), que analiza las diferencias individuales en el enfrentamiento de las adversidades, reporta que ninguno de los participantes con quienes trabajó ha tenido una vida sin dolor, esfuerzo y ansiedad y una de las conclusiones finales es que la adaptación efectiva al estrés es lo que nos permite vivir.

Es por lo anterior que las personas necesitan conocer los factores protectores y los posibles mecanismos para fortalecerlos, para que los procesos de vulnerabilidad / protección conduzcan a beneficios y no a desórdenes (Kotliarenco ,1995). 
Algunos de los factores protectores externos son la presencia de redes de apoyo, la valoración que las personas reciban del medio y las oportunidades para la participación, la contribución y el uso constructivo del tiempo (Kukic, 2002).

Ramsey y Blieszner (1999) muestran en su trabajo con mujeres adultas mayores espiritualmente resilientes, que la visión que las personas mayores más que problemas a ser resueltos, son recursos valiosos en sus familias y en la comunidad, contribuye al fortalecimiento de la resiliencia.

Al estudiar los factores protectores internos que constituyen los pilares de la resiliencia, se han identificado una serie de atributos personales. Estos se han agrupado en cuatro componentes que son según Suárez (1997):
a) Competencia social
b) Resolución de problemas
c) Autonomía
d) Sentido de vida

En Competencia social, se encuentran rasgos y características como:

- Mayor respuesta al contacto con y mayor generación de respuestas positivas en los otros.

- Capacidad para comunicarse y demostrar afecto o empatía.

- Sentido del humor.

- La moralidad, entendida como conciencia para extender el deseo personal de bienestar a toda la humanidad.

- Saber medir el temperamento propio y el de los otros (Grotberg, 1999; Suárez, 1997; Wolin y Wolin, 1993).

El componente Resolución de problemas incluye:

- La utilización de destrezas para planificar y para producir cambios en situaciones frustrantes

- Iniciativa

- Sentimiento de autosuficiencia

- Autoeficacia y autodisciplina

- Creatividad o capacidad para crear orden a partir del caos y el desorden (Suárez, 1997; Kotliarenco, 1995; Wolin y Wolin, 1993).

El componente Autonomía tiene como principal factor el sentido de la propia identidad, es decir, la habilidad para actuar independientemente. El locus de control interno y el sentido de 
poder personal son parte de este tercer componente, así como la capacidad para manejar sentimientos e impulsos propios (Grotberg, 1999; Suárez, 1997).

La capacidad para distanciarse de ambientes disfuncionales (distanciamiento adaptativo) y para saber pedir ayuda para utilizar los recursos de apoyo se ubican aquí, así como la iniciativa o disposición a exigirse y ponerse a prueba en tareas progresivamente más exigentes (Wolin y Wolin, 1993).

El cuarto componente, Sentido de vida, está constituido por ocho rasgos (intereses especiales, metas, motivación para el logro, aspiraciones educativas, optimismo, persistencia, fe y espiritualidad y sentido de propósito) que se relacionan con la intencionalidad que siguen las personas para alcanzar sus objetivos, la forma como se motivan y como evitan sentimientos de desesperanza (Kukic, 2002).

Antes de definir los rasgos del componente sentido de vida, que se convirtieron en los ocho focos de investigación del presente estudio, se puede mencionar que se ha encontrado una convergencia entre los factores que constituyen los pilares de la resiliencia y los que conducen al bienestar, el cual es producto de muchas decisiones vitales importantes. El bienestar se ha definido como un tono de ecuanimidad que se sostiene en el fondo, detrás de los eventos cotidianos más intensos, que puede sostenerse a pesar de y durante períodos de infelicidad; un navegar por la vida exitosamente, entendiéndose éxito como satisfacción en diversas dimensiones de la vida (Sheehy, 1987).

Otras características del adulto mayor sano con alto nivel de bienestar merecen ser mencionadas. Viorst (1992) resalta el sentirse valioso en lugar de verse como una víctima pasiva de su mundo interior y exterior. En otras palabras, considerarse un agente responsable y una fuerza determinante en su vida. Es importante señalar que el bienestar se logra activamente. Corey y Corey (1997) consideran que para alcanzarlo y mantenerlo la persona tiene que comprometerse con su propio bienestar. Enfatizan las elecciones que cada persona puede hacer, dentro de las limitaciones de cada situación, en los aspectos psicológico, social, intelectual y espiritual de su vida. Se trata, según ellos, de salud positiva, en oposición a la simple ausencia de síntomas debilitantes.

A continuación, se presenta una tabla adaptada con el perfil de la persona resiliente y se describen los rasgos relacionados con el componente de Sentido de vida, el cual es el eje de la presente investigación. 
El perfil de la persona resiliente sistematizado por Kukic (2002), se resume en la siguiente tabla.

\section{PERFIL DE LA PERSONA RESILIENTE}
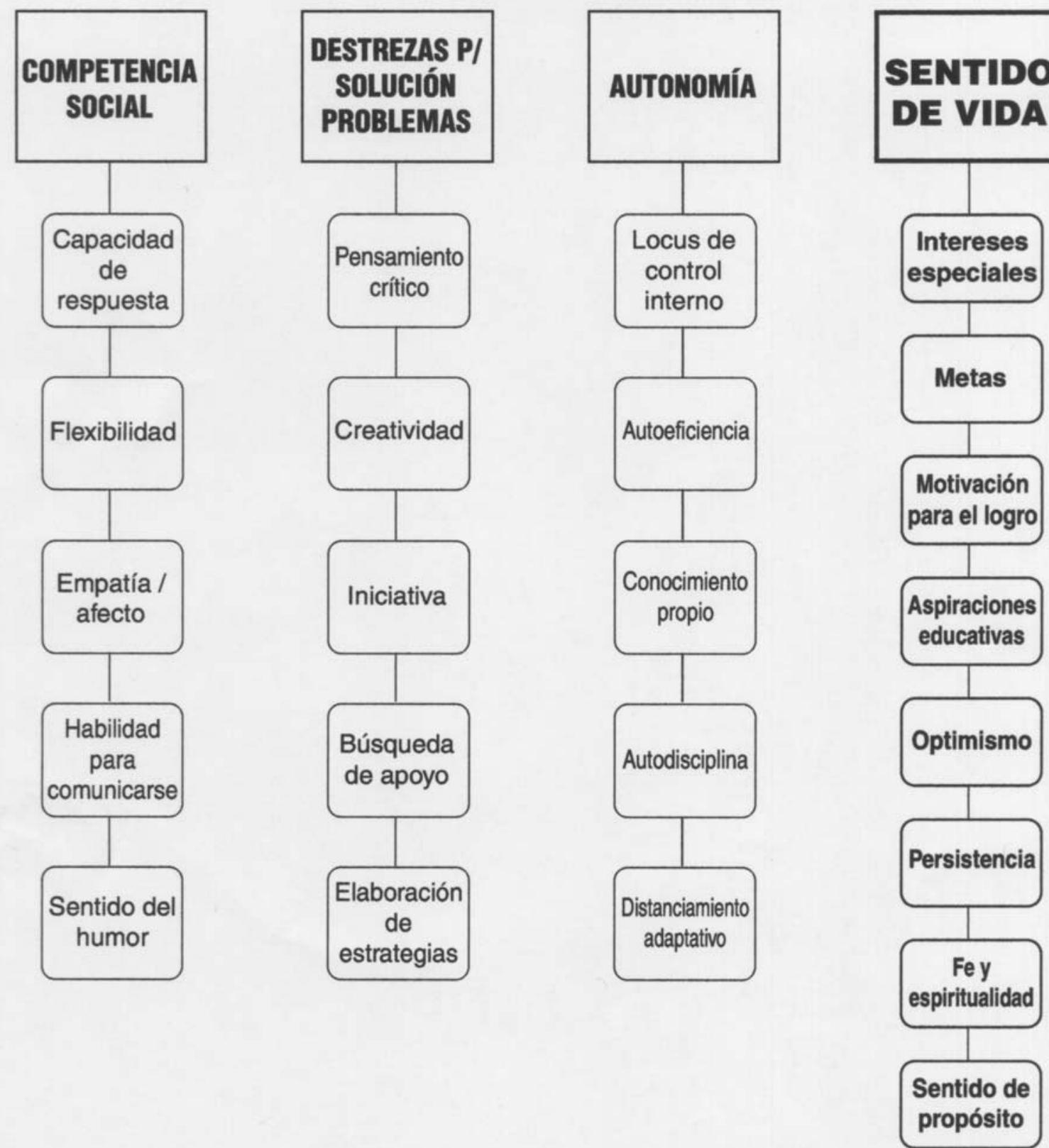


\subsection{RASGOS DE SENTIDO DE VIDA}

\subsubsection{Intereses Especiales}

Interés es aquella preferencia que se manifiesta a través de las actividades que la persona emprende durante su tiempo libre (pasatiempos), el contenido de sus lecturas favoritas, el éxito y el gusto en determinadas disciplinas escolares y en el trabajo (Casullo,1994). Las personas que tienen fuertes intereses y se pueden dedicar a ellos, manifiestan sentirse más satisfechas (Bessell y Kelly, 1977). Es lo que a la persona le llama la atención, a lo que le gusta dedicar tiempo, lo que le da placer. Tiene, de acuerdo con Rocabert (1995) un carácter motivador y reforzante.

El interés por las personas, cosas y otros elementos de la realidad, es un factor de motivación que ayuda a las personas a sentirse bien. El cultivo de los intereses cobra importancia durante todas las etapas del ciclo vital, así como cuando se tiene el desafío de enfrentar situaciones adversas (Erikson, 2000; Bisquerra, 2000; Raskind et al., 1999; Ramsey y Blieszner, 1999).

Para una mayor calidad de vida, es recomendable que los individuos tengan contacto con estímulos de aprendizaje en sus diversas dimensiones (física, afectiva, cognitiva, actitudinal, entre otras) (Corey y Corey ,1997).

\subsubsection{Metas}

Una meta es aquella finalidad que se persigue por medio de actividades específicas de cualquier índole. Su establecimiento da dirección y sentido a las acciones, lo que incrementa el rendimiento (Cuevas et al., 2001). La persona con dirección hacia metas organiza sus actividades en términos de lo que se ha propuesto y su cumplimiento. La meta en sí no es lo importante, sino que la persona se involucre en una actividad e invierta esfuerzos en el proceso (Cuevas et al., 2001). Cualquier meta puede dar significado a la vida, siempre que tenga objetivos claros, pasos para la acción y una manera de implicarse (Bonilla y Mata, 1998; Bisquerra, 2000).

Al estudiar longitudinalmente a personas que lograron un desarrollo exitoso a pesar de enfrentar limitaciones, Raskind, et al. (1999) encontraron que estas personas habían identificado metas temprano en la vida, lo que les dio dirección en el curso de su desarrollo. Asimismo, al definir sus metas, las habían visualizado como un proceso escalonado, es decir, por etapas. En sus vidas había evidencia de planeamiento pasado y futuro, y las metas planteadas eran alcanzables. 
El planteamiento de metas conlleva a valoraciones sobre la percepción del futuro más racionales que tienen que ver con lo que se ha llamado la intencionalidad, la cual se caracteriza por un impulso vital o entrega a una tarea a pesar de lo laboriosa o difícil que ésta pueda ser (Bühler citada por Abarca y Nassar, 1983).

\subsubsection{Motivación para el logro}

Es "un tipo de motivación orientada al éxito, frente a la motivación orientada hacia el temor al fracaso" (Castaño, 1995, p. 208), un impulso director que lleva a "esforzarse por mejorar o satisfacer un determinado criterio de excelencia" (Bisquerra 2000, p. 160). Este último autor anota dentro de las características de las personas dotadas de esta competencia el poseer una motivación fuerte para cumplir objetivos, el aprender a mejorar su desempeño y el afrontar objetivos desafiantes y asumir riesgos calculados. Se trata, entonces, del deseo de obtener éxito con una visión realista de las posibilidades. La motivación para el logro se refleja en las acciones concretas que realizan los individuos para alcanzar las satisfacciones que buscan. Por su parte Katz (1997) destaca la importancia de las experiencias de éxito para desarrollar este foco. Si en los diferentes ambientes se destacan las fortalezas, talentos y capacidades de las personas, se define la identidad alrededor de éstos y se desarrolla un sentido de dominio que favorece la motivación para el logro.

Las personas adultas mayores con alta motivación para el logro piensan que sus acciones tienen un valor, saben que pueden contribuir con el cambio y se esfuerzan por lograr sus objetivos. Todo esto les permite tener un propósito en la vida (Corrales, 1988). En la vejez, la motivación para el logro tiene la misma importancia que en otras etapas de la vida para la edificación de la resiliencia, a pesar de no saber cuánta vida se tiene por delante (Grotberg, 1999).

\subsubsection{Aspiraciones educativas}

Son los deseos que la persona adulta mayor tiene de aprender, para recibir un certificado o bien para enriquecer su vida mediante estudios constantes, periódicos u ocasionales. Este aprendizaje es "un proceso personal, pues nadie aprende por nadie, e implica un cambio de conocimientos, de conductas o de experiencias socioafectivas como resultado de la necesidad interna o de una demanda del medio ambiente" (Cazau, 2001, p. 3).

Las personas adultas mayores están conscientes de sus deseos educativos y se sienten motivadas para aprender, siempre y cuando ese aprendizaje satisfaga sus necesidades de experiencia e intereses. También se puede analizar el sentimiento general de competencia 
que tenga cada persona. Existe el riesgo de sentirse injustificadamente incompetente, debido a mensajes confusos que vienen de la sociedad o de la persona misma (Potter-Efron, 1998). Es importante tomar en cuenta las potencialidades de cada uno, su autoestima, sus valores, su relación con el ambiente, con las distintas fuerzas sociales y con sus deberes y derechos como ciudadanos. Esto les puede ayudar a alcanzar sus metas personales, familiares y laborales (Cazau, 2001; Graham, 1998).

Muchas personas adultas mayores continuarán "aprendiendo de la propia experiencia, haciendo cosas por ellos mismos, observando a otras personas e imitando o improvisando lo que ellos hacen" (Graham, 1998, p. 2).

\subsubsection{Optimismo}

Se entiende por optimismo la interiorización en los individuos, de la creencia que todo irá bien a pesar de los contratiempos y de las dificultades. El optimismo es una actitud que impide caer en la apatía, la desesperación o la depresión (Seligman, 1995).

Las personas optimistas piensan que los fracasos se deben a circunstancias que pueden cambiarse y, por lo tanto, que llegarán a triunfar si lo vuelven a intentar.

El optimismo puede aprenderse. La creencia de que se tiene el control de los acontecimientos de la vida y puede hacerse frente a los problemas, predispone a asumir riesgos y a desarrollar aptitudes y habilidades de las que se dispone (Bisquerra, 2000).

Para que una persona sea optimista debe hacer un análisis real de las situaciones, no exagerando lo positivo, pero tampoco anulándolo, no aislando lo negativo, pero tampoco quedándose en ello. A partir de esto, podría tener claro lo que quiere y cómo pretende llevarlo a cabo. Lo anterior le permitirá conseguir sus objetivos, a pesar de los obstáculos y contratiempos que se presenten (Diario El Tiempo, 2002).

Una persona mayor optimista cree que los acontecimientos positivos y felices se explican a través de las características que son permanentes (es decir, que seguirán ocurriendo en el tiempo) y generalizadas (o sea, que ocurren en diferentes ámbitos). Ésta asume la responsabilidad adecuada para lograr que los eventos buenos sucedan y, si ocurre algo negativo, lo considerará como temporal y específico de esa situación, además de mostrarse realista si es ella quien ha causado ese acontecimiento (Seligman, 1995).

Este factor de resiliencia se hace difícil entre más envejezca la persona, ya que se da cuenta que muchas cosas no salen bien. Los adultos mayores pueden enfrentar sufrimientos y adversidades y la habilidad para desafiarlos puede verse limitada (Erikson, 2000). Sin embargo, es posible cambiar las perspectivas que tiene la persona mayor acerca de las 
dificultades y cuando esto se logra se conservan los sentimientos de esperanza (Ramsey y Blieszner, 1999).

\subsubsection{Persistencia}

Persistente es la persona que continúa los esfuerzos a pesar de las adversidades, que describe las situaciones difíciles como experiencias de aprendizaje necesarias y que se concibe a si misma como alguien que no se da por vencido (Raskind et al., 1999). Para Seligman (1995), la persistencia es uno de los pilares de la productividad y brinda resistencia a la depresión a lo largo del ciclo vital. No se trata, según él, de que la persona adulta mayor no sienta tristeza, ansiedad o enojo. Lo que edifica la persistencia es precisamente continuar los intentos, a pesar de esos sentimientos desagradables, hasta acercarse lo más posible al dominio de lo deseado. Esto requiere la combinación de paciencia, tenacidad y la disposición a tolerar la frustración y la dificultad, hasta cumplir con el objetivo.

Algunas personas están constitucionalmente más inclinadas a este tipo de tenacidad que otras, pero todas pueden mejorarla y para todas es un elemento indispensable en su desarrollo como una persona productiva y para gozar de una mejor adaptación a los desafíos de la vejez (Grotberg, 1999; Bessell y Kelly, 1977).

\subsubsection{Fe y Espiritualidad}

Lo espiritual es la "capacidad, inherente a todo ser humano, de comportarse libremente y de forma responsable frente a las influencias internas y externas" (Noblejas, 2000, p. 23). Es la responsabilidad de aceptar la realidad de si mismo y de la propia vida, honrar el papel que se le ha confiado y llevarlo a cabo conscientemente. La espiritualidad "se refiere a aquellas actitudes, creencias y prácticas que animan (dan espíritu a) la vida de las personas" (Ramsey y Blieszner, 1999, p. 6). Proviene de muchas fuentes previsibles e imprevisibles, e implica asociarse y participar, comprometerse, cooperar, ser justo y respetar los derechos de todos los seres humanos (Edmund, citado por Ysern de Arce, 1999). Según Gardner (2001), lo espiritual se refiere a la capacidad de la especie para interesarse en asuntos trascendentales, sin que se trate de alcanzar una verdad final.

Siegel, citado por Corey y Corey (1997), describe la espiritualidad como la habilidad para encontrar paz y felicidad en un mundo con limitaciones y el sentir que se es imperfecto como persona, pero aceptable. Esta aceptación conduce a la creatividad y a la habilidad para amar (Fromm, 1974). 
La fe es un elemento de la estructura vital, como tal, hay condiciones que pueden quebrantarla y condiciones que pueden fortalecerla (Fromm, 1992). La persona con fe y espiritualidad tiene confianza, seguridad y esperanza dadas por convicciones o experiencias subjetivas, sean religiosas o no. La fe y la espiritualidad son formas de prepararse para que las adversidades resulten ser menos traumáticas y desorientadoras (Elkind, 1998).

\subsubsection{Sentido de propósito}

La identidad y el sentido de propósito no están presentes al nacer, más bien evolucionan a partir de las relaciones con otros y con la sociedad en un período histórico específico, con su composición única de eventos, valores y normas. El sentido de propósito es la intencionalidad o visión que las personas tienen hacia el futuro y se caracteriza por un impulso vital o entrega a una causa (Ramsey y Blieszner, 1999; Frankl, 1999; Bühler citada por Abarca y Nassar, 1983). El descubrimiento de este objetivo o esta misión es una tarea de toda la vida. No es algo que alguien nos puede decir o que descubrimos repentinamente (Daisaku, 1998).

El tener un sentido de propósito permite situarse más allá de los logros o derrotas del momento y ver las cosas en su debida perspectiva. Puede llenar cada instante de la vida con alegría, incluso en medio de circunstancias muy difíciles (Daisaku, 1998; Longaker, 1998).

Los adultos mayores que cada día valoran lo que realizan en su familia, su trabajo, su comunidad y en cualquier otro ámbito, tienen un sentido de logros alcanzados y una sensación de profunda satisfacción. Esto les permite tener un sentimiento de su propia dignidad y de su propio valor, de su condición de ser vivo y de su significación como ser humano único (Dyer, 1984).

\subsection{Papel de la Orientación para promover el sentido de vida en las personas adultas mayores}

La Orientación es una profesión de ayuda, consustancial a la Educación, que busca el bienestar del individuo. La labor en Orientación es educativa, de apoyo y situacional; trabaja con personas en forma individual, familiar y grupal (Gordillo, 1996). Asume que la persona es capaz de tomar decisiones responsables y con posibilidades de desarrollo. Esto implica que se han de rescatar y destacar las fortalezas que tienen los individuos, así como desarrollar nuevas habilidades para enfrentar sus vidas (Bonilla, 1993). Lo anterior les permitirá tomar conciencia de su proyecto de vida, el cual es "un proceso educativo que 
permite al ser humano lograr desde su concepción hasta la ancianidad, la actualización del proyecto total de sí mismo, de forma continua e ininterrumpida, pero de ninguna manera homogénea, y por ello, imposible de ser estandarizada" (Ludojoski, 1984, p.87).

La Orientación brinda asesoría a las personas en lo referente a las tareas del desarrollo que se enfrentan durante el ciclo vital, así como en la propuesta y evaluación de planes de vida en los ámbitos educativo, vocacional y personal-social (Corey, 1989; Rogers, 1984).

Para lograr lo anterior la persona profesional en Orientación se apoya en la identificación de metas, intereses, valores, habilidades y limitaciones personales, así como en la exploración de las condiciones y recursos que el medio provee.

Bonilla y Villarreal (1990, p. 92) plantean que la Orientación, “...es la ayuda integral que se facilita a los individuos para que desarrollen fortalezas humanas y manejen su propia vida, es decir, para que elaboren sus propios puntos de vista, tomen sus decisiones y asuman sus responsabilidades".

La persona profesional en Orientación que desee trabajar con adultas y adultos mayores promoviendo la construcción y clarificación de su sentido de vida, debe tener conocimiento de las teorías del desarrollo, de teorías y propuestas sobre sentido de vida, así como de los procesos de autoconocimiento e identidad, del contexto natural y sociocultural y del proceso de toma de decisiones, con el propósito de ayudarlos a asumir su existencia en la etapa y las situaciones vitales que estén experimentando (Escuela de Orientación y Educación Especial, 2000; Gordillo, 1996; Coto et al.,1991; Rogers, 1984). En esta investigación se trabajó sobre los rasgos de sentido de vida que han sido identificados por el enfoque de resiliencia.

Apoyar a la persona adulta mayor en la clarificación y construcción de su sentido de vida, le permitirá asumir la responsabilidad de encontrar respuestas satisfactorias a las tareas que la vida le asigna (Frankl ,1994).

\section{MÉTODO DE INVESTIGACIÓN}

Los principios teórico-metodológicos que guiaron esta investigación son los del paradigma cualitativo. Era importante tomar en cuenta los significados que las personas adultas mayores detallarán en relación con los rasgos de sentido de vida del enfoque de resiliencia. Interesó principalmente el significado y descripción que daban las personas mayores a cada uno de los rasgos de sentido de vida durante su vida y la forma como se manifiestan en la actualidad. 
Se utilizó la técnica de entrevista en profundidad, para conocer el proceso vivido por las participantes en relación con cada uno de los rasgos:

- Intereses especiales

- Metas

- Motivación para el logro

- Aspiraciones educativas

- Optimismo

- Persistencia

- Fe y espiritualidad

- Sentido de propósito

Con pequeñas modificaciones, se tomó como fundamento el modelo que presenta Antón de Schutter (1983) para la investigación cualitativa, organizado en cuatro etapas:

- Conocimiento global de la población mayor

- Preparación de las investigadoras

- Definición metodológica

- Contacto con la población mayor

1. Recolección de la información

2. Resultados y Análisis

La I Etapa se refiere al conocimiento global de la población y a la selección y contacto con el grupo por estudiar. En este caso se trabajó con ocho adultos mayores, con edades entre los 65 y 75 años de edad, 4 mujeres y 4 varones, del Área Metropolitana.

Los ocho participantes pertenecen al Grupo de Envejecimiento Integral de la Universidad de Costa Rica o a la Asociación Gerontológica Costarricense.

La II Etapa tiene que ver con la preparación de las investigadoras que se llevó a cabo por medio de estudio, capacitación y sensibilización respecto de la aproximación teórica conceptual y, en general del proceso investigador. Se definió el objeto de estudio, propósitos del mismo y se analizaron modos de abordarlo.

En la III etapa se seleccionaron los métodos por seguir y las investigadoras se prepararon para llevar a cabo el trabajo de campo.

La recolección de los datos se efectuó por medio de la entrevista en profundidad, se realizaron durante una sesión de entre una hora y hora y media cada una. En cada sesión se retroalimentó y fortaleció a los participantes con relación a lo que ellos expresaban, dándose un proceso educativo. 
La IV Etapa se refiere al trabajo de campo con las personas participantes. En esta etapa se debía establecer contacto con las personas mayores (se les llamó por teléfono para invitarles a participar en el estudio y se les visitó en las aulas en donde recibían cursos o talleres).

La mayoría de las entrevistas se realizaron en las casas de los participantes. En todos los casos la entrevista fue grabada y posteriormente transcrita.

Después de recoger la información obtenida por medio de las entrevistas, fue necesario agrupar los datos en un cuadro y tablas que respondieran a los objetivos formulados.

\section{INFORMACIÓN RECOPILADA Y ANÁLISIS}

Aunque se analiza la información obtenida a partir de cada uno de los rasgos de sentido de vida del enfoque de resiliencia, todos estos tienen relación entre sí.

\section{Intereses}

Los principales intereses que han dado significado a las personas participantes con edades entre 65 y 75 años en el grupo estudiado durante sus vidas en el caso de las mujeres: el trabajo, actividades en pro de la comunidad, actividades recreativas, la lectura y gusto por la Navidad. En lo que respecta a los varones: el deporte, el baile, la artesanía, escuchar a otras personas y la música.

En relación con los intereses que manifestaron las personas se pudo identificar que la intencionalidad ha estado centrada en vivir plenamente, mantener la independencia tanto económica como social, ayudar a otros y desarrollar talentos. Estas personas han realizado actividades que les permiten darse gusto a sí mismas, sentirse motivadas, sentirse bien consigo mismas, y que si bien dedicaban tiempo a sus intereses, en la actualidad el tiempo que dedican a éstos es más, ya que no tienen las múltiples ocupaciones que tenían en las etapas anteriores a la vejez (Ramsey y Blieszner, 1999).

Al final de la vida, se valora el haber podido identificar en etapas tempranas las actividades que les permitieran saber en qué querían invertir la energía, lo cual les permitiera la realización personal.

En síntesis, las personas entrevistadas han identificado y se han dedicado a algunos de sus intereses, lo cual les ha permitido, sentirse activas, motivadas, sentirse bien y lograr lo que Erikson (2000) menciona sobre la necesidad de tener intereses a lo largo de la vida que permitan tener una intención que les guíe. 


\section{Metas}

Entre las principales metas que plantearon las adultas mayores durante sus vidas, principalmente en el pasado, se pueden mencionar: capacitar a otras personas, la crianza de los hijos, transmitir la fe, la obtención de una casa y lograr tener un hogar estable. Por su parte los varones mencionan como sus metas el aprendizaje de un oficio, la adquisición de una casa, lograr integrar alguna organización y estudiar.

Todas las metas señaladas permiten decir que el sentido de vida de las personas del estudio parece ir más allá de lograr metas personales; se sienten responsables de necesidades sociales y familiares. Este impulso se ha convertido en el deseo de cumplir una especie de misión, que al lograrla provoca autoconceptos auténticos y esto les ayuda a que su autoestima y valor social sean también verdaderos y tengan por consiguiente sentimientos positivos respecto de si mismos y de su entorno (Bonilla y Rapso, 1997).

Se puede afirmar que las personas participantes en el estudio lograron aclarar su noción de mundo y del propio papel en él, han sentido la obligación por participar, por definir metas, que les permitieron descubrir lo que era fundamental, por qué luchar, siendo responsables de sus actos, ya que incorporaron una visión hacia el futuro.

Cabe destacar que este grupo de personas se caracterizan por estar activas, están integradas a grupos cuyo objetivo es precisamente que se mantengan productivos y vigentes, informados, y que establezcan redes de apoyo social para que permanezcan integrados a la sociedad. Sin embargo, es preocupante que la mayoría de las personas entrevistadas no lograron identificar metas en la actualidad, pese a que participan en actividades recreativas y educativas en los grupos a los que pertenecen, situación que debe provocar en las instituciones encargadas de generar políticas, programas y proyectos dirigidos hacia la población envejeciente, una reflexión, para poder favorecer estrategias que permitan a las personas adultas mayores, clarificar sus metas en la etapa de la vejez. Las metas en esta etapa podrían estar más centradas en ellos mismos y relacionarse con el conservar la dignidad, manejar la economía propia, lograr atención adecuada para su salud y participar en actividades útiles (Carnevalli y Patrick 1988).

\section{Motivación para el logro}

Las personas participantes identifican la motivación para el logro en las actividades que durante sus vidas han realizado. Las mujeres consideran que el estar activas les ha permitido estudiar, adquirir bienes materiales, tener buenas relaciones con los que les rodean y practicar algún deporte. Por su parte los varones reconocen que la motivación para 
el logro ha estado representada en la búsqueda del ascenso laboral, en el estudio para obtener mejor empleo o bien para aprender un oficio, en el involucrarse en actividades políticas y en el proponerse actividades que puedan realizar.

Se puede afirmar que las personas adultas mayores han estado activas y ocupadas, relacionándose con los que les rodean, han adquirido logros educativos en el pasado que les han permitido ser productivos y creativos, han tenido objetivos desafiantes para mejorar el desempeño y los han alcanzado. Han conseguido tener una visión positiva de si mismos por medio de acciones concretas para alcanzar la satisfacción que buscan, valoran y se sienten motivados con las actividades que realizan, esforzándose por mejorar un determinado criterio de excelencia y asumiendo compromisos calculados (Bisquerra, 2000).

Las labores que han realizado las personas participantes en el estudio les han permitido sentirse satisfechas y autosuficientes por vivir en un ambiente instituido por si mismas, ya que las actividades que realizan exigen el ejercicio y desarrollo de múltiples capacidades y virtudes, que a su vez perfeccionan a los que las desempeñan (Corrales, 1988).

La forma en que las personas mayores perciben la vida, la satisfacción o fracaso que experimenten, es siempre una respuesta personal que depende de la historia de cada una y de la forma en la que haya asumido su estilo de vida, ya que se envejece como se ha vivido. Si siempre les gustó cambiar, si fueron reflexivas sobre los eventos de su vida y se adaptaron a las circunstancias de la misma y lograron vivir con entusiasmo las tareas en cada etapa del desarrollo, la vida será entonces interesante (Bonilla y Rapso, 1997).

Para fortalecer el sentido de vida en lo que respecta a la motivación para el logro la persona adulta mayor debe mantenerse motivada mediante una evaluación positiva del pasado e incorporar nuevos papeles y actividades que reemplacen a los antiguos. Una vejez plena de sentido es aquella en la que se tiene una actitud contemplativa y reflexiva, reconciliándose con los logros y fracasos, y con los defectos. Debe lograrse la aceptación de uno mismo y aprender a disfrutar de los placeres que esta etapa brinda, lo que se obtiene más fácilmente si ha tenido preparación en las etapas anteriores.

La persona mayor autorrealizada tiene claridad de su sentido de vida, toma decisiones adecuadas, tiene valores y actitudes que muestran tolerancia, pero sobre todo tienen claridad de que la autorrealización es una búsqueda constante durante todo el ciclo vital (Grotberg, 1999). 


\section{Aspiraciones educativas}

Entre las principales aspiraciones educativas que plantearon las adultas mayores durante sus vidas se pueden mencionar: capacitarse sobre temas espirituales para su beneficio personal y para aportar a otros, la lectura, cursos para aprender a hacer diversidad de actividades como repostería, mecanografía, costura, entre otros. Además, se menciona el aprendizaje afectivo para aplicar en otros ámbitos. Por su parte los varones mencionan como sus aspiraciones educativas: estudiar para tener oportunidades laborales en otras zonas geográficas, aprender por medio de la lectura, de la observación, deseos de capacitación en la edad adulta, aprendizaje de otras generaciones por medio de la escucha o bien preguntando.

Los participantes del estudio pertenecen a una generación que en su mayoría no asistió al sistema educativo formal, sin embargo obtuvieron muchas capacitaciones que les permitieron y aun les permiten desenvolverse en la vida y enseñar a otros (Graham, 1998). Han sido aprendices activos teniendo como intencionalidad la educación permanente, en donde la persona es la única constructora de su propio conocimiento, y en la que la construcción se da solamente a través de su acción y su experiencia personal (Cazau, 2001). Lo más importante para el grupo del estudio es que están claros de sus deseos educativos y se sienten motivadas por aprender, siempre y cuando ese aprendizaje favorezca sus necesidades de experiencia e intereses (Potter-Efron, 1998).

Es importante resaltar los aprendizajes afectivos que manifiesta una de las participantes, lo cual podría hacer pensar que las personas adultas mayores podrían ser modelos para otras generaciones. Asimismo, cabe destacar que muchas de las personas participantes han logrado satisfacer sus deseos de aprender por medio de la observación o la comunicación verbal, con el propósito de lograr incorporar nuevos conocimientos y actitudes a sus vidas, mostrando respeto por los que les podían o pueden enseñar (Graham,1998).

En la actualidad la mayoría de las personas adultas mayores del estudio pertenecen a algún grupo que les facilita aprendizajes significativos para sus vidas, ya que pueden recibir cursos universitarios con personas de otras generaciones, capacitación acerca de cómo envejecer sanamente, sobre acondicionamiento físico, literatura, idiomas, artesanía, entre otros, logrando la adquisición de nuevos conocimientos.

\section{Optimismo}

Las personas participantes identifican el optimismo en la actitud que han tenido frente a acontecimientos significativos en sus vidas. Las mujeres lo perciben como la iniciativa 
constante en medio de cualquier situación de vida, así como el lograr un equilibrio, buscar apoyo en otros y el ver las características personales negativas como temporales y específicas. Por su parte, los varones lo consideran como un aprendizaje en medio de situaciones difíciles y el comportarse de acuerdo con sus principios, buscar alternativas de resolución y el ver las características personales negativas como temporales y específicas.

Se podría afirmar que las personas adultas del estudio han logrado durante sus vidas ser personas optimistas, que han enfrentado las experiencias difíciles como eventos que les permitieron algún aprendizaje, que al usar sus recursos personales han logrado el autocontrol y la toma de decisiones adecuada para lograr resolver los problemas. Han tenido una flexibilidad que les ha favorecido para adquirir la autoaceptación que el día de hoy les ayuda a tener gratitud por lo que tienen.

Estas personas consideran que el regirse por valores les ha fortalecido el optimismo, facilitándoles la interacción con los que les rodean, así como, el logro de sus metas. Se sienten satisfechos pues lo anterior les ha permitido mantener una actitud de lucha ante las circunstancias, lo que ha desencadenado en un proceso de bienestar (Bisquerra, 1998).

La mayoría de las personas mayores ha tenido la habilidad para enfrentar las modificaciones de su entorno y las situaciones experimentadas, renovándose y equilibrando las vicisitudes que se les han presentado en la vida, pudieron ejercer algún control sobre ellas, tomar la guía correcta y el compromiso, no evidenciando una visión pesimista de la vida.

\section{Persistencia}

Las personas adultas del estudio identifican la persistencia en las actividades que durante sus vidas han realizado. Las mujeres definen la persistencia como el aprendizaje que obtienen de las experiencias difíciles, así como el deseo de hacer bien las tareas que se proponen, lo cual les brinda satisfacción con lo que hacen. Se observa que al ser persistentes han logrado obtener satisfacciones y bienes materiales. Los varones por su parte también perciben las experiencias difíciles como fuentes de aprendizaje y presentan el deseo de hacer bien sus proyectos.

Se podría decir que las personas entrevistadas han realizado esfuerzos significativos como trabajar para obtener logros y dedicar tiempo a actividades que les permitieran un aprendizaje.

Cabe rescatar que estas personas han aprendido del error, no se han rendido, han tenido constancia, se han animado, sienten gusto por lo que han hecho y por lo que hacen; han aprendido de las experiencias adversas ( Raskind, et al., 1999). 
Las personas adultas mayores parecen haber tenido claro durante sus vidas su intencionalidad, han sido productivas y en la última parte de sus vidas están logrando la integridad, a partir de las fortalezas que han tenido. Siendo capaces de hacerle frente a la vida con dignidad a pesar de los fracasos y los sufrimientos que enfrentaron.

\section{Fe y Espiritualidad}

Las personas participantes identifican la fe y la espiritualidad como actitudes que han tenido frente a acontecimientos significativos en sus vidas. Las mujeres las ven como guías para respetar a los otros; mencionan la importancia de la autoaceptación y la espiritualidad como un apoyo. Por su parte los varones consideran que la fe y la espiritualidad les han guiado y apoyado, las perciben como medios que les han permitido tener valores que les han facilitado una relación adecuada con quienes interactúan.

Los participantes perciben la fe y la espiritualidad como fuentes de desarrollo personal que les han permitido fortalecer sus relaciones interpersonales por medio del respeto propio y el de los demás. Consideran que el haber sido espirituales les permite el día de hoy ver la vida con gratitud y como un aprendizaje en medio de las pérdidas, el sufrimiento y las alegrías. La fe y la espiritualidad han sido guías que les ha conducido durante sus vidas (Ramsey y Blieszner, 1999).

En las entrevistas realizadas las personas adultas mayores le dan significado a lo desconocido, opinan sobre la importancia de tener fe y espiritualidad como una afirmación de su concepción clarificada y de búsqueda de caminos hacia lo que es de interés del espíritu. La búsqueda de sentido de la vida está en los fundamentos emocionales y motivacionales que se expresan en la fe y la espiritualidad y éstas dan la experiencia de enlace e integración, de unidad y trascendencia de lo frecuente y ordinario que se asocia al bienestar general y al mayor significado de la vida, a pesar de los desafíos de la edad (Bonilla y Méndez, 2001).

Lo anterior había sido encontrado en estudios realizados por Bonilla y Rapso, (1997) y Bonilla y Méndez (2001), en los cuales se concluye que en las personas adultas mayores la intencionalidad fluctúa entre dos líneas que van de lo material a lo espiritual. La inversión material sería la que ellos hacen en sus familias y su propio desarrollo para realizar sus metas. La línea más espiritual es la del servicio a los demás, que asumen en algunos casos como una misión para reconciliarse con un ser superior y con ellos mismos. Podría entenderse esta intención como la integridad de la que habla Erikson (1970), que supera el 
amor a sí mismos por el amor a la especie humana, lo que les permite aceptar la vida que han vivido y superar el temor a la muerte.

En las personas adultas del presente estudio, se percibe la integridad como la madurez suficiente para valorar lo que se ha vivido desde una perspectiva realista, sin desesperarse sino aceptando lo que la vida ha sido. Con el paso del tiempo, el autoconocimiento es mucho mejor que en años anteriores; esto hace a su vez que se pierda poco a poco el interés por lo físico y que se piense en continuar hacia lo espiritual, lo cual hace que la persona tenga confianza, seguridad y esperanza dadas por las convicciones o experiencias subjetivas, sean religiosas o no (Elkind, 1998).

\section{Sentido de propósito}

Las adultas mayores mencionan que durante sus vidas han tenido sentido de propósito por medio del servicio y la transmisión de valores a otras generaciones. Por su parte los varones mencionan como su sentido de propósito el apoyo a sus familias, el enseñar a otros y el esforzarse en las labores que han tenido que realizar.

En las personas adultas mayores el sentido de propósito se encuentra en el apoyo, la enseñanza, el aportar conocimientos a las nuevas generaciones, dedicarse a actividades productivas a lo largo de la vida y la ayuda a otras personas, teniendo claridad de sus limitaciones. Estas personas, parecen haber encontrado la paz en un mundo imperfecto, el cual les ha permitido tener relaciones significativas (Elkind, 1998; Ramsey y Blieszner, 1999).

Se puede pensar que las personas del estudio lograron descubrir su misión o intención de vida, lo que les permitió situarse más allá de los logros o derrotas del momento y ver las cosas en su debida perspectiva, teniendo sentido de su propia dignidad y de su propio valor, de su condición de ser vivo y de su significación como ser humano único (Daisaku, 1998; Dyer, 1984).

La búsqueda interior que cada una de las personas ha hecho durante sus vidas les ha permitido clarificar su propósito de vida y llevarlo a la práctica. Se dieron cuenta que el "ideal de la vida humana depende de actitudes creativas, flexibilidad, exploración de alternativas, originalidad, capacidad y tolerancia" (Bonilla y Méndez, 2001, p. 9).

\section{CONCLUSIONES}

La investigación "Rasgos de sentido de vida del enfoque de resiliencia en personas adultas mayores de 65 a 75 años", ha permitido llegar a algunas conclusiones a partir de los objetivos planteados. 
Las ocho personas participantes del estudio (cuatro varones y cuatro mujeres) tienen edades entre 65 y 73 años, son funcionales e independientes, lo que les permite participar en grupos como el del Programa de Envejecimiento de la Universidad de Costa Rica y los de la Asociación Gerontológico Costarricense.

\section{Intereses}

Los principales intereses que han dado significado a las personas participantes con edades entre 65 y 75 años en el grupo estudiado durante sus vidas en el caso de las mujeres: el trabajo, actividades en pro de la comunidad, actividades recreativas, la lectura y gusto por la Navidad. En lo que respecta a los varones: el deporte, el baile, la artesanía, escuchar a otras personas y la música. La intencionalidad de las personas adultas mayores ha estado centrada en mantener la independencia tanto económica como social, ayudar a otros y en desarrollar talentos.

\section{Metas}

Entre las principales metas que plantearon las adultas mayores durante sus vidas, principalmente en el pasado, se pueden mencionar: capacitar a otras personas, la crianza de los hijos, transmitir la fe, la obtención de una casa y lograr tener un hogar estable. Por su parte los varones mencionan como sus metas el aprendizaje de un oficio, la adquisición de una casa, lograr integrar alguna organización y estudiar. Los participantes se dedican a satisfacer necesidades sociales y familiares, lo que les ha permitido tener sentimientos positivos respecto de si mismos y de su entorno. Es preocupante que las personas adultas mayores del estudio no identificaron metas claras en la actualidad.

\section{Motivación para el logro}

Las personas participantes identifican la motivación para el logro en las actividades que durante sus vidas han realizado. Las mujeres consideran que el estar activas les ha permitido estudiar, adquirir bienes materiales, tener buenas relaciones con los que les rodean y practicar algún deporte. Por su parte los varones reconocen que la motivación para el logro ha estado representada en la búsqueda del ascenso laboral, en el estudio para obtener mejor empleo o bien para aprender un oficio, en el involucrarse en actividades políticas y en el proponerse actividades que puedan realizar. Las personas mayores han estado activas y ocupadas, en interacción con los que les rodean y han adquirido logros que les han permitido ser activos y creativos. 


\section{Aspiraciones educativas}

Entre las principales aspiraciones educativas que plantearon las adultas mayores durante sus vidas se pueden mencionar: capacitarse sobre temas espirituales para su beneficio personal y para aportar a otros, la lectura, cursos para aprender a hacer repostería, costura, mecanografía, entre otros. Además, se menciona el aprendizaje afectivo para aplicar en otros ámbitos. Por su parte los varones mencionan como sus aspiraciones educativas: estudiar para tener oportunidades laborales en otras zonas geográficas, aprender por medio de la lectura, de la observación, deseos de capacitación en la edad adulta, aprendizaje de otras generaciones por medio de la escucha o bien preguntando. Los participantes del estudio pertenecen a una generación que en su mayoría no asistió al sistema educativo formal, sin embargo, obtuvieron diversas capacitaciones que les permitieron y aun les permiten desenvolverse exitosamente en la vida y enseñar a otros.

\section{Optimismo}

Las personas participantes identifican el optimismo en diferentes situaciones que han enfrentado. Las mujeres lo perciben como la iniciativa constante en medio de cualquier situación de vida, así como el lograr un equilibrio, buscar apoyo en otros y el ver las características personales negativas como temporales específicas. Por su parte los varones lo consideran como un aprendizaje en medio de situaciones difíciles y en comportarse de acuerdo con sus principios, buscar alternativas de resolución y el ver las características personales negativas como temporales y específicas. Estas personas han sido optimistas y han enfrentado las experiencias difíciles como eventos que les permitieron algún aprendizaje. Consideran que el regirse por valores les ha fortalecido el optimismo, facilitándoles la interacción con los que les rodean, así como el logro de sus objetivos.

\section{Persistencia}

Las personas adultas del estudio identifican la persistencia en las actividades que durante sus vidas han realizado. Las mujeres definen la persistencia como el aprendizaje que obtienen de las experiencias difíciles, así como el deseo de hacer bien las tareas que se proponen, lo cual les brinda satisfacción con lo que hacen. Se observa que al ser persistentes han logrado gratificaciones y la obtención de bienes materiales. Los varones por su parte también perciben las experiencias difíciles como fuentes de aprendizaje y presentan el deseo de hacer bien sus proyectos y no darse por vencidos ante alguna situación adversa. 
Cabe destacar que las personas han aprendido del error, no se han rendido y han sido constantes.

\section{Fe y Espiritualidad}

Las personas participantes identifican la Fe y la Espiritualidad como actitudes que han tenido frente a acontecimientos significativos en sus vidas. Las mujeres las han tenido como guía para respetar a los otros; mencionan la importancia de la autoaceptación y la espiritualidad como un apoyo. Por su parte los varones consideran que la Fe y la Espiritualidad les han guiado y apoyado, las perciben como medios que les han permitido tener valores que han facilitado una relación adecuada con quienes interactúan. Las personas entrevistadas consideran que el haber sido espirituales les permite el día de hoy ver la vida con gratitud y obtener aprendizajes en medio de las pérdidas, el sufrimiento y las alegrías.

\section{Sentido de propósito}

Las adultas mayores mencionan que durante sus vidas han tenido sentido de propósito por medio del servicio y la transmisión de valores a otras generaciones. Por su parte los varones mencionan como su sentido de propósito el apoyo a sus familias, el enseñar a otros y el esforzarse en las labores que han tenido que realizar. Estas personas han encontrado su sentido de propósito en el aportar conocimiento a las nuevas generaciones, dedicarse a actividades productivas a lo largo de la vida y la ayuda a otras personas, teniendo claridad de sus limitaciones. Se puede pensar que las personas del estudio lograron descubrir su misión o intención de vida.

Las personas participantes del estudio señalan que durante sus vidas contaron con algunas condiciones y herramientas que contribuyen con el desarrollo de los rasgos de sentido de vida del enfoque de resiliencia. Su participación actual en los programas ofrecidos por instituciones como la Universidad de Costa Rica y la Asociación Gerontológica les permite en la etapa de la vejez un fortalecimiento de su sentido de vida.

En síntesis, se podría concluir que las personas participantes del estudio han presentado durante sus vidas los diferentes rasgos de sentido de vida del enfoque de resiliencia, lo que les ha ayudado a tener una buena calidad de vida, adecuadas relaciones interpersonales, diversos logros y herramientas para enfrentar situaciones vitales. 


\section{A modo de recomendación}

Desde la disciplina de Orientación es necesario diseñar, ejecutar y evaluar estrategias sobre los rasgos de sentido de vida del enfoque de resiliencia dirigidas a diferentes poblaciones, que permitan promover en las personas la clarificación de la intencionalidad de sus vidas, para que así puedan llegar a las etapas posteriores con condiciones optimizadas para un adecuado bienestar.

\section{REFERENCIAS}

Abarca. S. y Nassar S. (1983). Psicología del adulto. San José, Costa Rica: Editorial Universidad Estatal a Distancia.

Bessell, H. y Kelly, T. (1977). The Parent Book. California: JALMAR PRESS.

Bisquerra, R. (2000). Educación emocional y bienestar. España: Editorial Praxis.

Bonilla, F.S. (1993). Orientación de las Poblaciones Abusadas. Revista de Ciencias Sociales, (59).

Bonilla, F. y Mata, A. (1998). Plenitud después de los 60: proyecto de toda una vida. Costa Rica: Editorial de la Universidad de Costa Rica.

Bonilla, F. y Méndez, A. (2001). Octogenarios y sentido de vida. Revista Anales en Gerontología, 3, (3), pp. 7-26.

Bonilla, F. y Rapso, M. (1997). Sentido de vida en personas mayores de 50 años. Revista Reflexiones, (59), pp. 29-43.

Bonilla, F y Villarreal, C. (1990). Concepto de Orientación: Evolución y Desarrollo en los Cursos de Práctica Supervisada. Revista de Educación, 14 (1), pp. 89-106.

Carnevalli, D. y Patrick, M. (1988). Tratado de Geriatría y Gerontología. México: Interamericana.

Castaño, C. (1995). Manual de asesoramiento y Orientación Vocacional. Madrid: Editorial Síntesis, S. A.

Casullo, M., et al. (1994). Proyecto de vida y decisión vocacional. Buenos Aires: Paidós.

Cazau, P. (2001). Andragogía. Recuperado el 15 de setiembre de 2003, de http://www.galeon.hispavista.com/pcazau/artdid-andra.htm.

Corey, G. (1989). Theory and Practice of Group Counseling. California: Brooks/Cole Publishing Company. 
Corey G. y Corey M. (1997). I Never Knew I Had a Choice. California: Brooks/Cole. Publishing Company.

Corrales, L. (1988). Los factores que influyen en la satisfacción laboral: El caso de tres organizaciones. Tesis de Licenciatura: Universidad de Costa Rica, Universidad de Costa Rica. Escuela de Orientación y Educación Especial, Costa Rica.

Coto, S. et al. (1991). Pautas de Interacción en Familias con un Miembro Alcohólico y Propuesta de Orientación Familiar Tesis de Licenciatura, Universidad de Costa Rica, Escuela de Orientación y Educación Especial, Costa Rica.

Cuevas, M., et al. (2001). Orientación Educativa 2. México: McGraw-Hill.

Daisaku, I. (1998). El sentido de tener un objetivo en la vida. Traducción de los textos publicados en la revista semanal Filipina Femenina Mirror en Sección: Relación Humana.

De Schutter, A. (1983). Investigación participativa: Una opción metodológica para la educación de adultos. México: CREPAL.

Diario El Tiempo. (2001). Optimismo. Recuperado el 26 de abril de 2003, de http://www.colegiobolivar.ed.co/high-school/10/Philosophy/Optimismo.htm

Dyer, W. (1984). El cielo es el límite. España: Grijalbo.

Elkind, D. (1998). All Grown Up and No Place to Go. Massachusetts: Addison-Wesley.

Erikson, E. (1970). Infancia y Sociedad. Argentina: Hormé.

Erikson, E. (2000). El ciclo vital completado. España: Paidós.

Escuela de Orientación y Educación Especial. (2000). Plan de Estudios de Bachillerato en Ciencias de la Educación con énfasis en Orientación. San José, Costa Rica: Facultad de Educación. Universidad de Costa Rica, Costa Rica.

Fabry, J. (1992). La búsqueda de significado. México: Fondo Cultura Económica.

Frankl. V. (1994). El hombre en busca de sentido. Barcelona: Herder.

Fromm, E. (1974). La revolución de la esperanza. México: Paidós.

Fromm, E. (1992). El corazón del hombre. México: Paidós.

Gardner, H. (2001). La inteligencia reformulada. España: Paidós.

Gordillo, M. (1996). Orientación y Comunidad. La Responsabilidad social de la Orientación. España: Alianza.

Graham, P. (1998). Aprendizaje de los Adultos. Recuperado el 22 de marzo de 2004, de http://www.aceproject.org/main/esanol/ve/vec01a01.htm

Grotberg E. (1999). Tapping Your Inner Strength. California: New Harbinger Publications. Volumen 4, Número 2, Año 200426 
Katz, M. (1997). On Playing a Poor Hand Well. Nueva York: WW Norton \& Company.

Kotliarenco, M. (1995). Una nueva mirada a la pobreza. Chile: Foro Mundial.

Kukic, S. (2002). Resilience Skills for Educators and Students: Teaching and Modeling Resilience. Annual Convention and Expo. United States of America: Council for Exceptional Children.

Longaker, C. (1998). Afrontar la muerte y encontrarle esperanza. México: Grijalbo.

Ludojoski, R (1984). Antropología: Educación del Hombre. Argentina: Guadalupe.

Maslow, A. (1976). The Farther Reaches of Human Nature. United States of America: An Esalen Book.

Noblejas, M. (2000). Palabras para una vida con sentido. Bilbao: Desclée de Brouwer.

Potter-Efron. (1998). Being Belonging Doing. Balancing Your Three Greatest Needs. California: New Harbinger Publications.

Ramsey, J., Blieszner, R. (1999). Spiritual Resiliency in Older Women. California: SAGE Publications.

Raskind, M. et al. (1999). Patterns of Change and Predictors of Success in Individuals with Learning Disabilities: Results From a Twenty-Year Longitudinal Study. Learning Disabilities Research \& Practice, 14(1).

Rocabert, E. (1995). Manual de Asesoramiento y Orientación Vocacional. Madrid: Editorial Síntesis.

Rogers, C. (1984). Orientación psicológica y psicoterapia. Madrid: Narcea.

Seligman, M. (1995). The Optimistic Child. Nueva York: HarperPerennial.

Sheehy, G. (1987). Crisis en la Edad Adulta. México: Grijalbo.

Suárez, N. (1997). Perfil del niño resiliente. Seminario Internacional sobre aplicación del Concepto de Resiliencia en Proyectos Sociales. Universidad Nacional de Lanús. Argentina: Colección Salud Comunitaria.

Thorson, J. (1995). Aging in a Changing Society. United States of America: Wadsworth.

Vaillant, G. (2000). Adaptation to Life. Massachusetts: Harvard University Press.

Viorst, J. (1990). Pérdidas necesarias. España: Muy Interesente.

Ysen de Arce, J. (1999). Inteligencia emocional en el adulto mayor. Chile: Seminario Internacional sobre la Tercera Edad. Universidad del Bio-Bio.

Werner, E., Smith, R. (2001). Journeys from Childhood to Midlife. Risk, Resilience, and Recovery. Nueva York: Cornell University Press. 
Wolin, S, Wolin, S. (1993). The Resilient Self: How Survivors of Troubled Families Rise Above Adversity. New York: Villard. 